\title{
INFLUENCE OF THE COMBINED DRY FRICTION ON THE DYNAMICS OF THE RIGID BALL MOVING ALONG TWO PARALLEL RAILS
}

\author{
ALEXEY A. KIREENKOV ${ }^{1,2}$ \\ ${ }^{1}$ Moscow Institute of Physics and Technology (State University), Institutskiy per. 9, Dolgoprudny, \\ Moscow Region, 141701, Russia, kireenkov.aa@mipt.ru \\ ${ }^{2}$ Institute for Problems in Mechanics of Russian Academy of Sciences, 119526, Prospect \\ Vernadskogo 101, korp.1, Moscow, Russia, kireenk@,ipmnet.ru, kireenk@,mail.ru
}

Key words: Combined Dry Friction, "Butterfly" Robot.

\begin{abstract}
The extended and improve version of the investigation described in [1-2] and the firstly presented in [3] is proposed in this work. Distinguish features of these investigations are applications of the theory of multi-component dry friction [4-16] in the problem of the ball rolling on boundaries of two identical frames. Father development of this model can be used in investigation of the dynamic of the a so-called "Butterfly" robot, consisting of two identical shaped plates rigidly placed parallel to each other on a small distance aimed at manipulating a ball that can freely roll on the plates' boundaries as on rails [17]. The difficult control systems considered in [18-20] are another engineering application of the combined dry theory. Following developed in previous investigation approach, the friction force and torque are computed by the integration over the contact area so that the exact dynamically coupled integral model accounting the relationship of all the components of friction is obtained. This exact model is replaced by approximated analytical model [1-2] which is completely satisfy to all analytical properties of integral model as function kinematics parameters without increasing the number of coefficients.
\end{abstract}

\section{INTRODUCTION}

The problem of the ball rolling on boundaries of two identical frames is interested applications of the theory of the combined dry friction. It was on the example of the study of the classical problem about the dynamics of a ball on a rough plane that the necessity of creating this theory and its further development were demonstrated. The distinguishing feature of this theory is presenting a more convenient form of the coupled friction models for the problems of solids dynamics. One of the main assumptions of this theory is the validity of the classical Amonton-Coulomb law in differential form for a small element of the area inside the contact spot of the rubbing solids. Then, the net vector of the friction force and torque are computed by the integration over the contact area and presents the exact coupled integral models of the combined dry friction.

It is worth explain the used of the term "Exact integral model" because any model can not be exact, because it is only an approximation to a real phenomenon. This notion is used in the sense that, after the initial assumptions about the validity of Coulomb's law in classical and 
generalized differential form and general properties of the normal contact distributions inside of contact spot, all other computations, from a mathematical point of view, are being made exactly, without the use of approximate methods. Thus, after writing expressions for the differentials of the dry friction principal vector and torque, all subsequent transformations are exact results, reflecting the nature of the phenomenon.

The integral model gives a good description of the dry friction effects in the case of combined kinematics, but is inconvenient to be used in problems of dynamics, because it is required to calculate multiple integrals in the right-hand sides of the equations of motion. To avoid calculating double integrals, the exact model is replaced by an approximated analytical model [4-16], which fully satisfies all the analytical properties of the integral model as a function of kinematic parameters without increasing the number of coefficients.

The proposed dry friction models enables as well to describe the relationship between force and kinematical characteristics by smooth analytical functions over the entire range of angular and linear velocities as to take into account the more realistic representation about normal contact stresses distribution and differential characteristics of the friction law. The approximate models preserve all analytical properties of the models based on the exact integral expressions and correctly describe the behavior of the net vector and torque of the friction forces and their first derivatives at zero and infinity.

Moreover, the models coefficients are numbers that can be identified from experiments [21-24]. Consequently, these models may be considered as phenomenological models of the combined dry friction

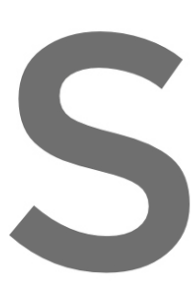

\section{Development of the} is not only of indepan describe more correctly t. called "Butterfly" robo rigidly placed paralle to each other on a small

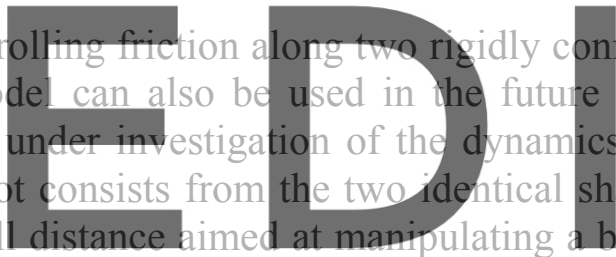
e in order to
ics of the so-
shaped plates
ball that can freely roll on the plates' boundaries as on rails.

Register for free at https//www.scipedia.com to download the version without the watermark 2 PROBLEM STATEMENT AND BASIC ASSUMPTIONS

A free rolling of a heavy uniform ball of radius $R_{b}$ (Fig.1.b) along the two identical rails rigidly placed parallel to each other (Fig.1.a) is considered (Fig.1). It is supposed that the ball is involved in the complex motion: simultaneous rolling, spinning and sliding along the rails. 


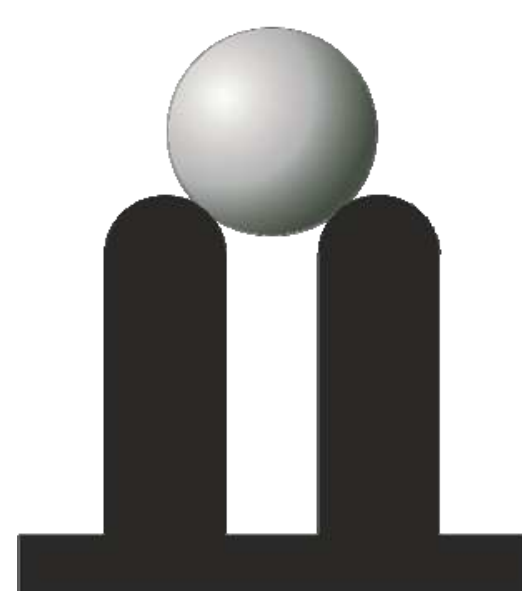

(a)

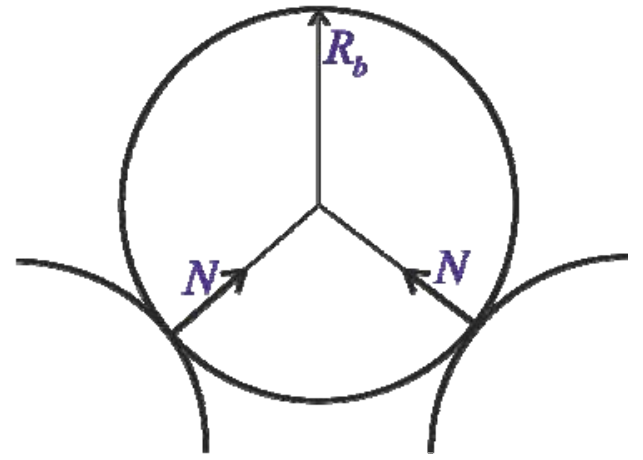

(b)

Figure 1. Scheme of contact

The ends of the rails are assumed to be rounded, which allows us to assume that the contact surfaces of the rubbing bodies are locally spherical and the contact spots have circle form. Therefore, normal reactions at the point of contact are directed along the radiuses of the ball. Under these assumptions, the distributions of the normal contact stresses inside of contact spots at the absence of ball motion are can be described by the Hertz law [19-20]:
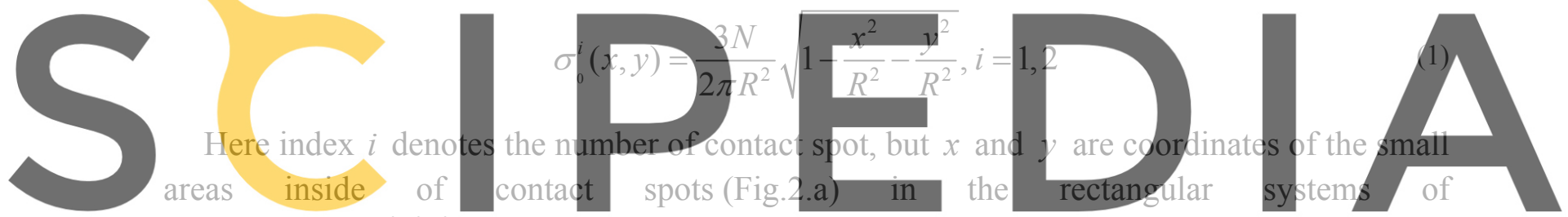

coordinates $\left\{O_{i} \mathrm{e}^{\mathrm{i}} \mathrm{e}^{\mathrm{i}} \mathrm{e}^{\mathrm{i}}\right\}, i=1,2$. Origins of the considered coordinates systems are hardly

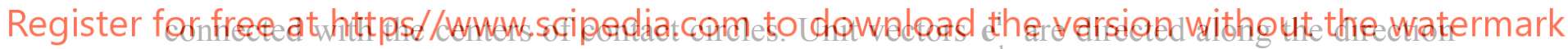

of the ball sliding, parallel to the rails. Unit vectors $\mathrm{e}_{3}^{\mathrm{i}}$ are perpendicular directed to the plane of contact spot parallel to ball radius. Unit vectors $\mathbf{e}_{2}^{\mathrm{i}}$ are directed perpendicularly to the direction of the ball sliding and complement vectors $\mathbf{e}_{1}^{\mathbf{i}}$ and $\mathbf{e}_{3}^{\mathbf{i}}$ up to the right triple.

The resultant vectors of friction forces and torque of friction can be presented in the considered coordinates systems by the following formulas:

$$
\mathbf{F}_{\mathbf{i}}=F_{\|}^{i} \mathbf{e}_{1}^{\mathbf{i}}+(-1)^{i} F_{\perp}^{i} \mathbf{e}_{2}^{\mathbf{i}}, \quad \mathbf{M}_{\mathbf{i}}=M_{\|}^{i} \mathbf{e}_{1}^{\mathbf{i}}+(-1)^{i} M_{\perp}^{i} \mathbf{e}_{2}^{\mathbf{i}}+(-1)^{i} M_{C}^{i} \mathbf{e}_{3}^{\mathbf{i}}
$$

The difference in signs in formulas (2) is connected with difference in direction of the ball rotation relative to the contact spots.

Another main assumption is that the Amonton-Coulomb law in differential form holds for the small surface element $d S_{i}$ in the interior of the contact spot. According to this law, the differentials of the resultant vector $d \mathbf{F}^{\mathrm{i}}$ and the moment of friction $d M^{i}$ with respect to the contact spot center are determined by the following formulae [5-15]: 


$$
d \mathbf{F}=-f \sigma \frac{\mathbf{V}}{|\mathbf{V}|} d S, d M_{C}=-f \sigma \frac{\mathbf{r} \times \mathbf{V}}{|\mathbf{V}|} d S, \mathbf{V}=(v-\omega y, \omega x), \mathbf{r}=(x, y)
$$

where $f$ is the coefficient of friction; $\mathbf{r}=(x, y)$ is the position vector of an elemental area in the interior of the contact spot with respect to its center Fig. $2 . \mathrm{a} ; v$ is velocity of the relative sliding inside of contact spot, but $\omega$ is the component of the angular velocity of the ball rotation respectively to the contact spot plane. The velocity $\omega$ can be considered as velocity of the contact spot rotation relatively to the ball. Its perpendicular directed to the contact spot in the direction the ball radius. In addition, index $i$ in formulas (3) is omitted for briefly of writing.

\section{DRY FRICTION MODEL}

There are well known results from the theory of elasticity [10-11, 14-15] that tangent stresses lead to shift in the symmetric diagram of the normal contact stresses in the direction of the instantaneous sliding velocity $v$ or in the rolling direction Fig2.b.

To use these results in the dynamics problems, it is proposed the simple asymptotic representations for the contact stresses distributions based on their general properties [1-14]:

$$
\sigma_{1}(x, y)=\sigma_{10}\left(1+k_{x} x / R+k_{y} y / R\right)
$$
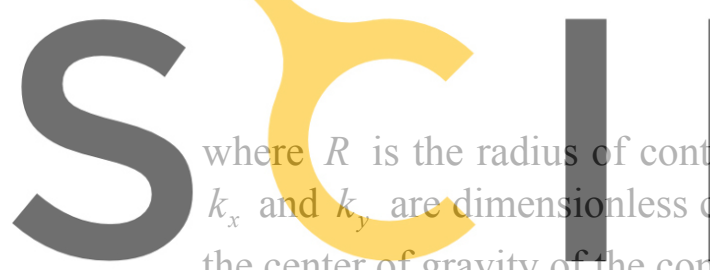

the center of gravity of the
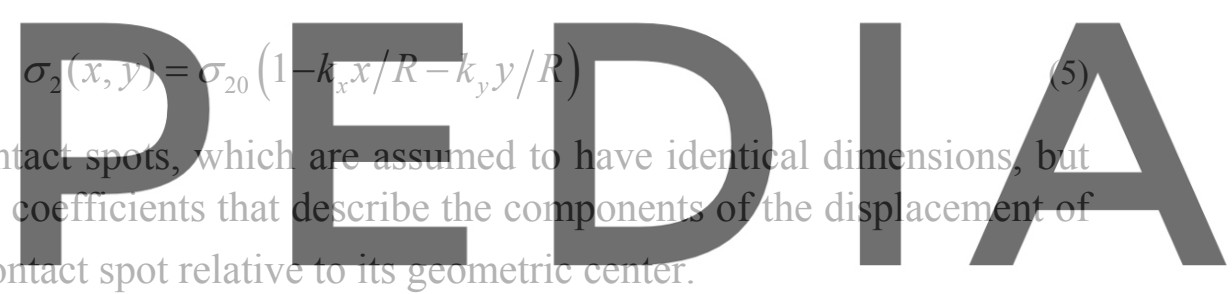

The difference in signs in formulas (4)-(5) is connected as in formula (2) with difference in

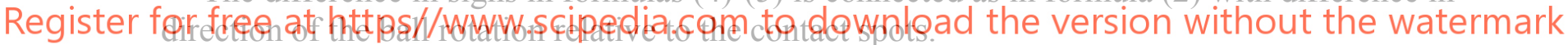

Presence of the simultaneously sliding and rolling in the different directions leads only to

summanization of the corresponded coefficients, which can be used on the base of the results of theory of elasticity or procedure developed in [5-15].

The displacement of the contact spot gravity results to the appearance of the rolling moment $\mathbf{M}_{\mathrm{r}}^{i}$ parallel to the contact plane whose projections parallel $\left(M_{\|}\right)$and perpendicular $\left(M_{\perp}\right)$ to direction of the relative sliding can be determined by the simple formulas from [14-15].

$$
\mathbf{M}_{\mathbf{r}}^{\mathbf{i}}=M_{\|}^{i} \mathbf{e}_{1}^{\mathbf{i}}+M_{\perp}^{i} \mathbf{e}_{2}^{\mathbf{i}}, i=1,2
$$






(a)



(b)

Figure 2. Kinematics inside the contact spot.

Integration of the corresponded differentials over the contact spot yields the resultant vectors $\mathbf{F}^{\mathrm{i}}$ of the friction force and torques $\mathbf{M}_{\mathrm{C}}^{\mathrm{i}}=(-1)^{i} M_{C} \mathbf{e}_{3}^{\mathrm{i}}$ :

$$
\mathbb{F}=-f \iint \sigma(x, y) \frac{\mathbf{V}}{|\mathbf{V}|} d x d y, \quad \mathbf{M}_{\mathbf{C}}=-f \iint \sigma(x, y) \frac{\mathbf{r} \times \mathbf{V}}{|\mathbf{V}|} d x d y,
$$


force) and the normal (lateral friction force) to instant velocity of sliding and the module of

Register for free at https//www.scipedia.com to download the version without the watermark

the formulas from [5-15]:

$$
\begin{aligned}
& F_{\|}=f \iint_{G} \frac{(v-\omega y)\left(1+k_{y} y / R\right) \sigma_{0}(x, y) d x d y}{\sqrt{\omega^{2}\left(x^{2}+y^{2}\right)+v^{2}-2 \omega v y}}, F_{\perp}=\frac{k_{x} f}{R} \iint_{G} \frac{\omega x^{2} \sigma_{0}(x, y) d x d y}{\sqrt{\omega^{2}\left(x^{2}+y^{2}\right)+v^{2}-2 \omega v y}} \\
& M_{C}=f \iint_{G} \frac{\left(\omega\left(x^{2}+y^{2}\right)-v y\right)\left(1+k_{y} y / R\right) \sigma_{0}(x, y) d x d y}{\sqrt{\omega^{2}\left(x^{2}+y^{2}\right)+v^{2}-2 \omega v y}}, G=\left\{(x, y): x^{2}+y^{2} \leq R^{2}\right\}
\end{aligned}
$$

These doubles integrals (8) can be calculated in elementary functions for Hertz distribution of the normal contact stresses [14-15].

The exact integral models gives a good description of the dry friction effects in the case of combined kinematics, but is inconvenient to be used in problems of dynamics, because it is required to calculate multiple integrals in the right-hand sides of the equations of motion.

Approximated model [5-12] based on the analytical properties of the integral model as a function of kinematic parameters allowed to avoid this inconvenience without increasing the number of coefficients. Coefficients of this model are numbers that can be easy calculated [512] or identified from experiments [21-23] in more complicated cases. This fact permits use 
results of previous investigation directly in considered problem. Thus, the dry friction model which is described the interaction between ball and rails can be represented in following form:

$$
\begin{aligned}
& M_{C}=\frac{M_{0} u}{\sqrt{u^{2}+m v^{2}}}, F_{\|}=\frac{F_{0} v}{\sqrt{v^{2}+a u^{2}}}, F_{\perp}=\frac{\mu F_{0} u}{\sqrt{\left(u^{2}+b v^{2}\right)}} \\
& M_{\|}=-M_{r} \frac{\Omega_{y}}{\Omega_{r}}, M_{\perp}=-M_{r} \frac{\Omega_{x}}{\Omega_{r}}, M_{r} \equiv s k_{r} N
\end{aligned}
$$

Coefficients of this model were calculated in previous works [5-15], but, as above, index $i$ is omitted for briefly of writing.

\section{ACKNOWLEDGEMENTS}

These investigations were supported by the RFBR, grant 20-08-01120 and partly by the state program of the scientific research works No.: AAAA-A20-120011690138-6.

\section{REFERENCES}

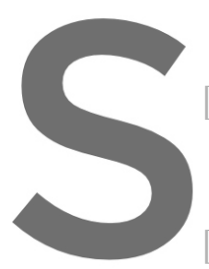

[1] A. Kireenkov, D Boundaries of Two Rails, in: WCCM-ECCOMAS2020.



Nonlinear

Systems

(2021)

AIP

Conference

Proceedings,

2343:120001.

Register for freelathteps/PwWw.scipedia.com to download the version without the watermark

[4] Kireenkov, A.A., Ramodanov, S.M. Combined Dry Friction Models in the Case of Random Distribution of the Normal Contact Stresses Inside of Contact Patches, 8th

International Conference on Coupled Problems in Science and Engineering, COUPLED PROBLEMS 2019 (2021):176-182.

[5] Kireenkov, A.A., Zhavoronok, S.I., Nushtaev, D.V. On tire models accounting for both deformed state and coupled dry friction in a contact spot. Computer Research and Modeling (2021) 13(1):163-173. (DOI: 10.20537/2076-7633-2021-13-1-163-173).

[6] Kireenkov, A.A., Zhavoronok, S.I. Anisotropic Combined Dry Friction in Problems of Pneumatics' Dynamics, Journal of Vibrational Engineering and Technologies (2020) 8 (2):365-372. (DOI: 10.1007/s42417-019-00140-1).

[7] Kireenkov, A.A. Improved friction model of the aviation tyre contact with the landing strip. IFAC-PapersOnLine (2018) 51(2) :890-894. (DOI 10.1016/j.ifacol.2018.04.027)

[8] Kireenkov, A.A., Nushtaev, D.V., Zhavoronok, S.I. A new approximate model of tyre accounting for both deformed state and dry friction forces in the contact spot on the background of the coupled model. MATEC Web of Conferences (2018) 211(08003). (DOI 10.1051/matecconf/201821108003)

[9] Kireenkov, A.A., Zhavoronok, S.I. Implementation of analytical models of the anisotropic 
combined dry friction in problems of pneumatics' dynamics. MATEC Web of Conferences (2018) 211(08004). (DOI 10.1051/matecconf/201821108004)

[10] Kireenkov, A.A., Zhavoronok, S.I. Coupled dry friction models in problems of aviation pneumatics dynamics. International Journal of Mechanical Sciences (2017) 127:198-203. (DOI 10.1016/j.ijmecsci.2017.02.004)

[11] Kireenkov, A.A. Improved theory of the combined dry friction in problems of aviation pneumatics dynamics. Proceedings of the 7th International Conference on Coupled Problems in Science and Engineering, COUPLED PROBLEMS 2017 (2017):1293-1298.

[12] Zhavoronok, S.I., Kireenkov, A.A. On the effect of the anisotropic dry friction and the deformed state of tires on the shimmy initiation. Proceedings of the 7th International Conference on Coupled Problems in Science and Engineering, COUPLED PROBLEMS 2017 (2017):216-226.

[13] Kireenkov, A.A. Further development of the theory of multicomponent dry friction. Proceedings of the oth International Conference on Coupled Problems in Science and Engineering, COUPLED PROBLEMS 2015 (2015):203-209.

[14] Kireenkov, A.A. Coupled model of sliding and spinning friction, Doklady Akademii Nauk (2011) 441(6): 750-755.

[15] Kireenkov, A.A. Coupled models of sliding and rolling friction, Doklady Akademii Nauk (2008) 419(6):759-762.

[16] Kireenkov, A.A. Three-dimensional model of combined dry friction and its application in

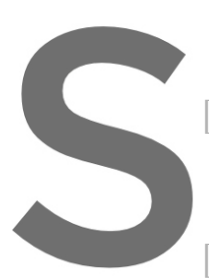
non-holonomic mechanics. Proceedings of ENOC-2005, Eindhoven, Netherlands, August 7-12 (2005):571-577.
Surov, M., Shiriaev, A. and ets., Case st
and orbital stabilization of one-diredional
-IEEE International Conference on Robotice

18] Ramodanov, S.M., Kireenkov, A.A. Con


the presence of friction. Proceedings of the 7 th International Conference on Coupled



[19] Semendyaev, S.V. Solid system with two massive eccentrics on a rough plane: rotational case. IFAC-Papers OnLine (2018) 51(2):884-889.

[20] Semendyaev, S.V. General case of movement of solid system with two massive eccentrics on a rough plane. Proceedings of the VIII International Conference on Coupled Problems in Science and Engineering (COUPLED PROBLEMS 2019) (2019):183-191 (ISBN: 978-84-949194-5-9).

[21] A. Kireenkov and S. Zhavoronok, A Method of Parameters Identification for the Coupled Dry Friction Model for Pneumatic Tires, in: WCCM-ECCOMAS2020. (www.scipedia.com/public/Kireenkov Zhavoronok 2021a)

[22] Kireenkov, A.A., Zhavoronok S.I. Numeric-Analytical Methods of the Coefficients Definition of the Rolling Friction Model of the Pneumatic Aviation Tire, 8th International Conference on Coupled Problems in Science and Engineering, COUPLED PROBLEMS 2019 (2021):204-212.

[23] Kireenkov, A.A., Semendyaev, S.V., Filatov, V.V. Experimental Study of Coupled TwoDimensional Models of Sliding and Spinning Friction. Izv. Akad. Nauk. Mekh. Tverd. Tela. (2010) 6:192-202. 